\title{
Evaluation of MIF as a Diagnostic Biomarker in Cases with Ankylosing Spondylitis Ibrahim Tharwat Abdelal ${ }^{1}$, Mirvat Abdel Hameed Eltoukhy ${ }^{1}$, Ahmed Mohammed Baraka ${ }^{2}$, Mohammed Abdelrahman Ibrahim ${ }^{1}$ \\ Departments of ${ }^{1}$ Rheumatology and Rehabilitation and ${ }^{2}$ Clinical Pathology, Faculty of Medicine, Zagazig University, Zagazig, Egypt \\ *Corresponding Author: Mohammed Abdelrahman Ibrahim, Mobile: (+20)01024683929, Email: franshiskoo@gmail.com
}

\begin{abstract}
Background: Ankylosing spondylitis (AS) has been afflicting humankind as far back as ancient Egypt. Macrophage migration inhibitory factor (MIF) appears to have the unique ability to drive both inflammation and new bone formation and could play an important role in the pathogenesis of AS.
\end{abstract}

Objective: The study aimed to evaluate MIF as a diagnostic biomarker in AS.

Patients and Methods: This study was conducted on 42 subjects; 21 AS cases and 21 controls. Patients were subjected to full medical history, general and musculoskeletal examinations, laboratory investigations including serum MIF level, sacroiliac X-ray, sacroiliac MRI with the Ankylosing Spondylitis Disease Activity Score (ASDAS) for disease activity.

Results: Regarding gender of the patients, the majority (71.4\%) of patients were males; while (28.6\%) were females. We found that; the mean disease duration of AS patients was $(15.05 \pm 6.69)$ years; while the mean ASDAS score was $(2.78 \pm 0.68)$. We also found that, $(71.4 \%)$ of AS patients received NSAIDs, (95.2\%) received diseasemodifying anti-rheumatic drugs (DMARDs), (57.1\%) received tumour necrosis factor inhibitors (TNFi). Regarding imaging findings in our study, (61.9\%) of AS patients had sacroiliitis in sacroiliac X-ray while (100\%) of AS patients had sacroiliitis in sacroiliac MRI.

Conclusion: Serum MIF levels are elevated in AS patients. ROC-curve analysis showed that the best cutoff point of MIF was (>41.3) with area under curve $(0.625)$, with fair accuracy $(57.1 \%)$, sensitivity $(80 \%)$ and specificity $(50 \%)$.

Keywords: Biomarker AS, Evaluation, MIF.

\section{INTRODUCTION}

Ankylosing spondylitis (AS) has been afflicting humankind as far back as ancient Egypt. It was during the 1800s that the classical description of AS was made. Throughout the 1900 s, further understanding about the disease was established, including its hereditary nature. The disease is recognised as part of the spondyloarthropathy group of rheumatic diseases. These include psoriatic arthritis, reactive arthritis, and arthritis associated with inflammatory bowel disease. These conditions share similar clinical features and an association with human leukocyte antigen (HLA)B27 (1).

AS is not just limited to the spine; the peripheral joints can be affected, and organs such as the eyes, heart, and lungs can be involved. Patients can also complain of systemic symptoms such as fatigue or weight loss. There is a high risk of osteoporosis and vertebral fractures. Chronic pain and immobility can lead to patients experiencing depression and anxiety. There is a socio-economic burden as patients may be unable to work, either due to their symptoms or a workplace that may not be adapted for people with arthritis. Thus, it is important to recognise that this is a multisystem disease and the clinician should be wary of focusing purely on spinal symptoms ${ }^{(2)}$.

Definite diagnosis can be delayed as radiographic changes of sacroiliitis occur late in the disease process. Patients who present without radiographic changes are described as having non-radiographic axial spondyloarthritis (nr-axSpA). Classification criteria have been updated to include the diagnosis of nr-axSpA. It should be noted that certain treatments, such as infliximab and interleukin (IL)-17 inhibitors, are not approved for nr-axSpA according to the Assessment of SpondyloArthritis International Society-European League against Rheumatism (ASAS-EULAR) 2016 recommendations, but other biologic drugs are approved. Overall, however, it is argued that only the single term of AS should be used to encompass the disease ${ }^{(3)}$.

Macrophage migration inhibitory factor (MIF) appears to have the unique ability to drive both inflammation and new bone formation and could play an important role in the pathogenesis of AS. Serum MIF levels could be considered a predictive of progressive spinal damage in AS patients ${ }^{(4)}$.

Also, Fiorillo et al. (5) in his article about ankylosing spondylitis and related immune-mediated disorders, reported that, CD74 is the receptor of the macrophage migration inhibitory factor (MIF), which is involved in the pathogenesis of AS and mediates its pro-inflammatory functions and mediated erosive process over time.

The study aimed to evaluate migration inhibitory factor as a diagnostic biomarker in ankylosing spondylitis. 


\section{PATIENTS AND METHODS}

This study was carried out in outpatients and inpatients clinics at Rheumatology and Rehabilitation Department, Faculty of Medicine, Zagazig University Hospitals and Rheumatology Department, Kobry El- Kobba Military Hospital and Maadi Military Hospital

Assuming that MIF level in AS patients is $31.0 \pm 11.4$, and in control group $22.1 \pm 8.3$, at confidence level $95 \%$, power $80 \%$, so, total sample size is 42 (21 in every group) calculated by open Epi.

Group 1; 21 patients with ankylosing spondylitis (AS) who were diagnosed according to modified New York criteria for AS ${ }^{(\boldsymbol{})}$.

Group 2; 21 Healthy volunteers (age and sex matched with the patients group) served as controls.

Inclusion criteria: Patients with ankylosing spondylitis (AS) who met the modified New York criteria for AS ${ }^{(6)}$.

Tools and instruments used in data collection: Demographic and clinical characteristics of patient's records including age, sex, clinical, family history, history of drugs intake, age at disease onset and disease duration.

Type of the study: Case control study.

\section{Examination:}

- General examination.

- Systemic examination.

- Locomotor examination:

- Peripheral joints examination: Assessment of patient's "Ankylosing Spondylitis Disease Activity Score (ASDAS)" "(7). ASDAS combines five disease activity variables, resulting in one single score with better truth (validity), enhanced discriminative capacity and improved sensitivity to change as compared to single-item variables.

Laboratory investigations for patients and controls including: Complete blood count (CBC). Erythrocyte sedimentation rate (ESR). C-reactive protein (CRP). Random blood sugar. Human leukocyte antigen (HLA-B27) ${ }^{(\boldsymbol{8})}$.
Imaging: Plain X-ray sacroiliac joints: widening, narrowing, erosion, ankylosing. MRI sacroiliac joints: on MRI, findings to consider include both active inflammatory lesions (primarily bone marrow edema) and structural lesions (e.g. bone erosions, new bone formation, sclerosis and fat infiltration).

\section{Serum MIF by enzyme-linked immunosorbent assay (ELISA) ${ }^{(4)}$. \\ Ethical considerations:}

The study design approved from the Institutional Review Board (IRB) of Zagazig University Hospitals. The World Health Organization (WHO) and the Declaration of Helsinki recommendations were followed, in terms of protecting the rights and well-being of the studied people. The nature of the present study and laboratory or radiological procedures was explained to all participants. Consent was obtained from all participants. At the end of the study, all patients were informed about the results of the examinations performed and received appropriate recommendations, and treatment.

\section{Statistical Analysis}

Data entry, processing and statistical analysis was carried out using MedCalc ver. 18.2.1 (MedCalc, Ostend, Belgium). Tests of significance (Mann-Whitney's, Chi square tests, and ROC Curve analysis) were used. Data were presented and suitable analysis was done according to the type of data (parametric and non-parametric) obtained for each variable. Quantitative data were expressed as mean \pm SD (Standard deviation). Independent samples t-test was used to compare between two independent groups of normally distributed variables (parametric data). $\mathrm{P}$ value $<0.05$ was considered significant.

\section{RESULTS}

There is statistically non-significant difference between the studied groups regarding age or gender (Table 1).

Table (1): Comparison between case and control groups regarding demographic characteristics

\begin{tabular}{|l|c|c|c|c|}
\hline \multirow{2}{*}{$\begin{array}{c}\text { Demographic } \\
\text { characteristics }\end{array}$} & \multicolumn{2}{|c|}{ Groups } & \multicolumn{2}{|c|}{ Test } \\
\cline { 2 - 5 } & AS group & Control group & \multirow{2}{\mathbf{2}}{$/ \mathbf{t}$} & $\mathbf{P}$ \\
\cline { 2 - 5 } & $\mathbf{N = 2 1 ( \% )}$ & $\mathbf{N = 2 1 ( \% )}$ & & \\
\hline $\begin{array}{l}\text { Gender: } \\
\begin{array}{l}\text { Female } \\
\text { Male }\end{array}\end{array}$ & $\begin{array}{c}6(28.6) \\
15(71.4)\end{array}$ & $\begin{array}{c}6(28.6) \\
15(71.4)\end{array}$ & 0 & \\
\hline $\begin{array}{l}\text { Age (years): } \\
\text { Mean } \pm \text { SD } \\
\text { Range }\end{array}$ & $\begin{array}{c}38.29 \pm 6.48 \\
26-53\end{array}$ & $\begin{array}{c}37.24 \pm 8.93 \\
20-55\end{array}$ & 0.435 & 0.666 \\
\hline
\end{tabular}

There is statistically significant difference between the studied groups regarding ESR (significantly higher in AS group) (Table 2). 
Table (2): Comparison between the studied groups regarding routine laboratory data

\begin{tabular}{|c|c|c|c|}
\hline \multirow[t]{3}{*}{ Laboratory data } & \multicolumn{2}{|l|}{ Groups } & \multirow[t]{3}{*}{$\mathbf{P}$} \\
\hline & AS group $(n=21)$ & Control group $(n=21)$ & \\
\hline & Mean \pm SD & Mean \pm SD & \\
\hline Hemoglobin(g/dl) & $11.93 \pm 1.37$ & $12.84 \pm 1.51$ & $0.047 *$ \\
\hline Platelet $\left(10{ }^{3} / \mathrm{mm}^{3}\right)$ & $195.19 \pm 36.76$ & $189.14 \pm 25.81$ & 0.541 \\
\hline $\operatorname{TLC}\left(10^{3} / \mathrm{mm}^{3}\right)$ & $6.02 \pm 1.44$ & $6.59 \pm 1.56$ & 0.314 \\
\hline ESR (mm/hour) & $31.75 \pm 3.2$ & $9.83 \pm 2.18$ & $<0.001 * *$ \\
\hline CRP (mg/dl) & $16.37 \pm 3.24$ & $12.88 \pm 3.31$ & 0.159 \\
\hline
\end{tabular}

There is statistically significant difference between the studied groups regarding MIF (significantly higher in AS group) (Table 3).

Table (3): Comparison between the studied groups regarding MIF value

\begin{tabular}{|l|c|c|c|c|}
\hline \multirow{3}{*}{ Laboratory data } & \multicolumn{2}{|c|}{ Groups } & \multicolumn{2}{c|}{ Test } \\
\cline { 2 - 5 } & AS group (n=21) & Control group (n=21) & Z & p \\
\cline { 2 - 5 } & Mean \pm SD & Mean \pm SD & & \\
\hline MIF $(\mathrm{ng} / \mathrm{dl})$ & $43.56 \pm 5.68$ & $11.37 \pm 3.16$ & -5.548 & $<0.001^{* *}$ \\
\hline
\end{tabular}

About $61.9 \%$ of patients had sacroiliitis by $\mathrm{X}$ ray, while $100 \%$ of patients had sacroiliitis by MRI (Table 4).

Table (4): Radiological characteristics of the studied patients with AS

\begin{tabular}{|l|c|c|}
\hline \multicolumn{1}{|c|}{ Radiological characteristics } & N=21 & \% \\
\hline X-ray: & 8 & 38.1 \\
Normal & 13 & 61.9 \\
Sacroiliitis & 21 & 100 \\
\hline MRI sacroiliac: & & \\
Sacroiliitis & & \\
\hline
\end{tabular}

There is non-significant relation between MIF levels and clinical manifestations, age, gender or disease duration of the studied patients (Table 5).

There is non-significant relation between HLA-B27 and MIF among the studied patients (Table 5).

Table (5): Relation between MIF and HLA-B27 among the studied patients

\begin{tabular}{|c|c|c|c|}
\hline \multirow{2}{*}{ HLA-B27 } & MIF (ng/d) & \multicolumn{2}{|c|}{ Test } \\
\cline { 2 - 4 } & Mean \pm SD & Z & p \\
\hline Absent $(8$ cases $)$ & $39.42 \pm 6.7$ & -0.29 & 0.772 \\
Present $(13$ cases $)$ & $46.11 \pm 19.1$ & & \\
\hline
\end{tabular}

The best cutoff of MIF was $\geq 41.3$ with area under curve 0.625 , sensitivity $80 \%$, specificity $50 \%$, positive predictive value $33.3 \%$, negative predictive value $88.9 \%$ and accuracy $57.1 \%$ (p>0.05) (Figure 1). 


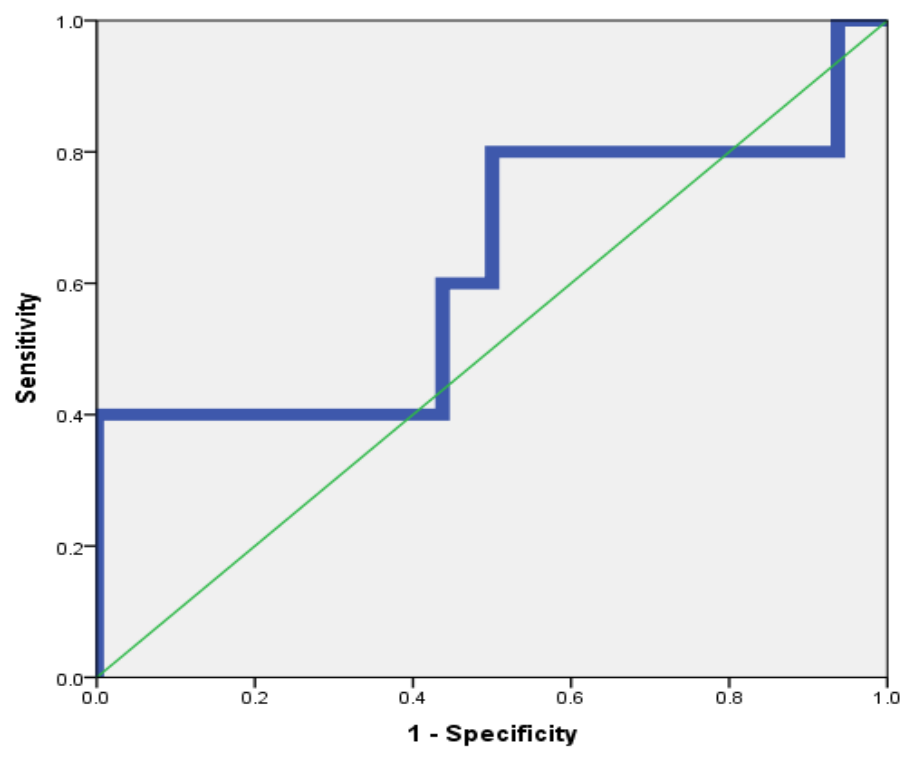

Figure (1) ROC showing performance of MIF.

\section{DISCUSSION}

Regarding clinical data in our study, we found that; the mean age of AS patients was (38.29 \pm 6.48 ) years and the mean age of healthy individuals was $(37.24 \pm 8.93)$ years. Regarding gender of the patients, the majority $(71.4 \%)$ of patients were males; while $(28.6 \%)$ were females.

Comparative study between the 2 groups also revealed non-significant difference as regards HLA-B27 ( $p>0.05)$. Our results came in agreement with Kozaci et al. ${ }^{(9)}$, who reported that, the comparison of the study participants according to HLA-B27 revealed no significant difference ( $p$ > $0.05)$.

There was significant positive correlation between Ankylosing Spondylitis Disease Activity Score (ASDAS), erythrocyte sedimentation rate (ESR) and migration inhibitory factor (MIF) among the studied patients. This is consistent with Ranganathan et al. ${ }^{(4)}$ which concluded that MIF appears to have the unique ability to drive both inflammation and new bone formation and could play an important role in the pathogenesis of AS. MIF not only promotes inflammation, but also triggers osteoblastic activity, suggesting a novel pathogenic role for this pleiotropic cytokine in AS agreed with our findings ${ }^{(10)}$.

Comparison between the 2 groups also revealed non-significant difference as regards all the laboratory variables. Our results came in harmony with Kozaci et al. ${ }^{(9)}$, who reported that, there was non-significant difference between the AS patients and the control subjects regarding hemoglobin level, platelet count and total leucocytic count ( $\mathrm{p}>0.05)$.

Regarding the acute phase reactants, there was a highly significant increase in ESR levels in AS group compared to control group. On the other hand Kozaci et al. ${ }^{\left({ }^{(9)}\right.}$ reported that, the ESR levels were significantly higher in the AS patients than in the controls. Our results also were consistent with results of De Vries et al. ${ }^{(11)}$ that stated that ESR, was significantly associated with the ankylosing spondylitis over 3 months, and the association with ESR was the strongest.

Regarding MIF levels, there was a highly significant increase in MIF levels in AS groups compared to control group. Our results agreed with Ranganathan et al. ${ }^{(4)}$ who reported that MIF levels were significantly elevated in AS patients compared to healthy controls. Also, Onuora ${ }^{(12)}$ demonstrated that serum levels of MIF were raised in patients with AS as compared with healthy individuals and Aydogan Baykara et al. ${ }^{(13)}$ reported that MIF levels are shown to be higher in AS patients compared to controls.

Regarding imaging findings in our study, $61.9 \%$ of AS patients had sacroiliitis in sacroiliac joints x-ray while $100 \%$ of AS patients had sacroiliitis in sacroiliac joints MRI.

Our results showed non-significant relation between disease activity (ASDAS ESR) and TNFi. This also agreed with results of Heinonen et al. ${ }^{(14)}$ which was conducted to describe the effectiveness and drug survival of tumor necrosis factor (TNF) inhibitors in the treatment of AS and to analyze the effect of concomitant treatment with conventional disease-modifying anti-rheumatic drugs and concluded that TNF inhibitors are equipotent in the treatment of AS; however, ETN (Etanercept) and ADA (adalimumab) were found superior to IFX (infliximab) in drug survival. The use of SSZ 
(sulfasalazine) improves treatment continuation. But our results disagreed with the study of Pedersen $\boldsymbol{e t}$ al. (15), which was conducted to evaluate responsiveness of the Ankylosing Spondylitis Disease Activity Score (ASDAS) in a 1-year follow-up study of patients with axial spondyloarthritis treated with tumor necrosis factor $\alpha$ inhibitors and found that ASDAS demonstrates construct validity and high responsiveness during treatment with $\mathrm{TNF} \alpha$ inhibitors in patients with $\mathrm{SpA}$.

\section{CONCLUSION}

Serum MIF levels are elevated in AS patients. ROC-curve analysis showed that the best cutoff point of MIF was (>41.3) with area under curve (0.625), with fair accuracy $(57.1 \%)$, sensitivity (80\%) and specificity $(50 \%)$.

\section{REFERENCES}

1. Taurog J, Chhabra A, Colbert R (2016): Ankylosing spondylitis and axial spondyloarthritis. N Engl J Med., 374(26): 2563-74.

2. Adrovic A, Barut K, Sahin S et al. (2016): Juvenile spondyloarthropathies. Current Rheumatology Reports, 18 (8): 55.

3. Van der Heijde D, Ramiro S, Landewé R et al. (2017): Update of the ASAS-EULAR management recommendations for axial spondyloarthritis. Ann Rheum Dis., 76(6): 978-91.

4. Ranganathan V, Ciccia F, Zeng F et al. (2017): Macrophage migration inhibitory factor induces inflammation and predicts spinal progression in ankylosing spondylitis. Arthritis and Rheumatology, 69(9): 1796- 806.

5. Fiorillo M, Haroon N, Ciccia F et al. (2019): Editorial: Ankylosing spondylitis and related immune-mediated disorders. Front Immunol., 10:1232-36.

6. Van der Linden S, Valkenburg H, Cats A (1984): Evaluation of diagnostic criteria for ankylosing spondylitis. A proposal for modification of the New York criteria. Arthritis Rheum., 27(4):361- 8.

7. Machado P, Landewé R, Braun J et al. (2012): MRI inflammation and its relation with measures of clinical disease activity and different treatment responses in patients with ankylosing spondylitis treated with a tumour necrosis factor inhibitor. Annals of the Rheumatic Diseases, 71(12): 2002-5

8. Caffrey M, James D (1973): Human lymphocyte antigen association in ankylosing spondylitis. Nature, 242:121-26.

9. Kozaci L, Sari I, Alacacioglu A et al. (2010): Evaluation of inflammation and oxidative stress in ankylosing spondylitis: a role for macrophage migration inhibitory factor. Modern Rheumatology, 20(1): 34-9.

10. Haroon $N$, Inman $R$, Learch $T$ et al. (2013): The impact of tumor necrosis factor a inhibitors on radiographic progression in ankylosing spondylitis. Arthritis Rheum., 65: 2645-54.

11. De Vries M, van Eijk I, van der Horst-bruinsma I et al. (2009): Erythrocyte sedimentation rate, C-reactive protein level, and serum amyloid A protein for patient selection and monitoring of anti-tumor necrosis factor treatment in ankylosing spondylitis. Arthritis Care \& Research, 61(11): 1484-90.

12. Onuora S (2017): Spondyloarthropathies: MIF drives inflammation and bone formation in AS. Nature Reviews Rheumatology, 13(8): 451-455.

13. Aydogan Baykara R, Tuzcu A, Omma A et al. (2019): Evaluation of serum thiol/disulfide homeostasis in patients with ankylosing spondylitis by a novel method. North Clin Istanb., 6(4):348-354.

14. Heinonen A, Aaltonen $K$, Joensuu $J$ et al. (2015): Effectiveness and drug survival of TNF inhibitors in the treatment of ankylosing spondylitis: a prospective cohort study. The Journal of rheumatology, 42(12): 2339-46.

15. Pedersen S, Sørensen I, Hermann K et al. (2010). Responsiveness of the Ankylosing Spondylitis Disease Activity Score (ASDAS) and clinical and MRI measures of disease activity in a 1-year follow-up study of patients with axial spondyloarthritis treated with tumour necrosis factor $\alpha$ inhibitors. Annals of the Rheumatic Diseases, 69(6): 1065-71. 